\title{
The domain of dependence fusion of low-altitude and slow-speed dim target detection
}

\author{
Hongwei Zhang ${ }^{1, a}$, Chenyu $\mathrm{Liu}^{1, \mathrm{a}}$ and Juntao $\mathrm{Ma}^{1, \mathrm{a}}$ \\ ${ }^{1}$ Mechanical Engineering College, Shijiazhuang 050003, China; \\ a406861173@qq.com
}

Keywords: Correlation region integration, Beam alignment, Clutter simulation, Cross cumulant.

\begin{abstract}
Aiming at the difficulty that regular single radar is very hard to detect land and sea clutter in echo of low-altitude and slow-speed dim target, it considers about to apply two radars receiving and dispatching respectively for radar echo's domain of dependence integration. According to the characteristic of weak correlation of clutter and strong correlation of target under different radar carrier frequency system, it simulates Rayleigh distribution which has a better matching with land and sea clutter, by means of zero memory nonlinearity method. In order to overlap echo of two radars in domain of dependence to improve signal to clutter ratio of output signal and to be in favor of detecting low-altitude and slow-speed dim target, it accumulates echo through calculating second order and three order of cross-cumulant of echo signal.
\end{abstract}

\section{Introduction}

With the development of modern war measures, nowadays various countries start to conduct the research of low-altitude penetration so that low-altitude penetration, integrated electronic interference, stealth target and anti-radiation missile have become the "four main threats" to monostation radar[1]. Furthermore, the detection of low-altitude and slow-speed dim target is also a technological problem recognized in the world. There are two major problems for regular monostatic radar detecting low-altitude and slow-speed dim target. The first one is strong clutter interference. Owing to low flight of object, a strong land and sea clutter should be mixed in echo, and RCS of these low-altitude and slow-speed target is only $0.05 \mathrm{~m}^{2} \sim 0.1 \mathrm{~m}^{2}$ [2]. Therefore, effectively restrain of clutter becomes one of the key of detecting this type of object. The second problem is lacking of observation time[3].Low-altitude penetration object often adopts terrain following tactic when attacking, that is to say, to shelter by rational utilization of landform causes that radar is very difficult to effectively observe the low-altitude penetration object for a long time. As a result of low resolution capability of Doppler, it is hard to effectively test targets.

However, people are beginning to push the limits of only applying monostation radar gradually along with the development of radar's new system and technology. Currently, integrated using of multistatic radar has increasingly become a new system of radar in modern warfare. Nevertheless, the majority of multistatic radar is fusing information based on information fusion in decision-making level and other high levels to detect an object at present, which leads to loss of partial information in the original response signal[4]. On the contrary, information fusion on the basis of echo signal in signal level will cause less loss of partial information. In particular, regular radar with narrower bandwidth is usually responsible for monitoring object, whereas information integrated utilization of multistatic radar has more practical significance on the task of monitoring object. Researches indicate that radar with different frequency bands has a weak correlation with clutter in echo and a strong correlation with the object in terms of a slowly moving target[5].As a consequence, it is feasible to calculate and accumulate via cross cumulant of corresponding echo pulse of two radars, and subsequently to identify the object due to larger cross cumulant of existing target's range cell and less cross cumulant of non-existent target's range cell. In this study, it establishes a mode of bistatic radar observing object under adjacent station-distribution, and enables the bistatic radar to achieve approximate simultaneous observation and correspond the number of echo pulse through the 
processing of beam alignment and pulse synchronization to the bistatic radar. Moreover, it calculates and accumulates pulse-to-pulse cross cumulant according to the characteristic of a strong correlation of radar target echo and a weak correlation of clutter, and improves signal-to-clutter ratio of echo that is in favor of detecting low-altitude and slow-speed dim target.

\section{Definition and nature of cross cumulant}

In accordance with statistical signal processing theory[6], the definition of K-order cumulant is:

$$
C_{x_{1} x_{2} X_{3} \ldots x_{k}}\left(t_{1}, t_{2}, t_{3} \ldots t_{k-1}\right)=\operatorname{cum}\left[X_{1}(n), X_{2}\left(n+t_{1}\right), X_{3}\left(n+t_{2}\right) \ldots X_{k}\left(n+t_{k-1}\right)\right]
$$

Hereinto, $X_{k}(n)$ is a real-valued stochastic process, and $n=0, \pm 1, \pm 2 \ldots, \mathrm{k}=1,2,3 \ldots$, and $t_{1}, t_{2} \ldots t_{k-1}$ are time delay. It would be called as cross cumulant between stochastic processes when there is more than one stochastic process.

Equally, cross cumulant possesses nature as followed:

(1) Cross cumulant owns semi-invariability. If stochastic processes $\left\{X_{k}\right\},\left\{Y_{k}\right\}$ are mutually independent, then:

$$
\operatorname{cum}\left[X_{1}+Y_{1}, X_{2}+Y_{2}, X_{3}+Y_{3} \ldots X_{k}+Y_{k}\right]=\operatorname{cum}\left[X_{1}, X_{2}, X_{3} \ldots X_{k}\right]+\operatorname{cum}\left[Y_{1}, Y_{2}, Y_{3} \ldots Y_{k}\right]
$$

(2) If stochastic process $\left\{X_{k}\right\}$ can be divided into two groups or multi-groups of statistically independent random variables, then their K-order cumulant is 0 .

Based on two features discussed above, it is easy to find that cross cumulant will be 0 theoretically, only if one group of the random variable is independent with other random variables.

As mentioned earlier, in corresponding moment clutter echo has a weak correlation and target echo has a strong correlation, while the estimated value of the target echo cross cumulant should be greater than the estimated value of clutter echo cross cumulant, under different frequency band radar illumination. Consequently, signal to clutter ratio of signal output can be improved according to output dependence that is in favor of succeeding detection of object.

\section{Correlative fusion procedure of bistatic radar}

(1) Conduct processing of beam alignment and impulsive synchronization to echo of bistatic rada $r$ with the purpose of two radars achieving approximate simultaneous observation object and corres-ponding echo pulse.

(2) Capture an appropriate distance of range bin from individual pulse of two radars respectively after the processing. If assume that each radar receives $\mathrm{N}$ echo pulses, each echo's range bin is $\mathrm{M}$, sampling number of each range bin is n, and there is no overlap between range bins, then envelope echo signal sampling of two radars is set as:

$$
\begin{aligned}
& E_{c h o_{1}}{ }^{N, M}=S_{1}{ }^{N, M}+C_{1}{ }^{N, M}+E_{1}{ }^{N, M} \\
& E_{c h o_{2}}{ }^{N, M}=S_{2}{ }^{N, M}+C_{2}{ }^{N, M}+E_{2}{ }^{N, M}
\end{aligned}
$$

Hereinto, $S, C, E$ represent target echo, clutter and noise component in echo respectively, and $N, M$ represent the Mth range bin in the Nth echo impulse. Subsequently, sample of second-order and third-order cross cumulant calculation can be represented as:

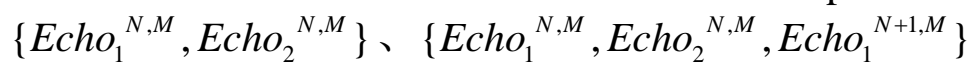

To calculate cross cumulant of range bins of two radars' corresponding pulse at the same position, we can obtain a $N \times M$ dimensional cross-cumulant value matrix, and ultimate cross-cumulant value vector by accumulating cross-cumulant value matrix of all pulses after processing:

$$
A=\left[c_{1}, c_{2}, c_{3} \ldots c_{M}\right]
$$

With respect to the vector above, it can be draw that correlation of clutter and noise will be relatively weak and thereby its cross cumulant output is relatively less if there is no object of range bin, while cross cumulant output of range bin with object should be greater than cross cumulant output of range 
bin without object. As a result, detection of an object can be conducted through the differences of cross cumulant output value.

\section{Pulse alignment in neighboring station-distributed radar observation model}

Depending on the mentioned above, precondition of echo cross-cumulant calculation is to satisfy synchronous scanning of two radars' beams, as well as one-to-one correspondence of echo pulse. At the condition of neighboring station distribution, spacing between two radars can be ignored compared to distance between radar and object, and consequently two radars can be considered as observing in the same place.

As shown in figure when distance between radar 1 and radar 2 is very close, it can be approximately regarded as two radars are observing an object at the same place. And at this moment,differences between radar beam and echo pulse mainly involve as followed:

(1) Scanning cycle of radar antenna;

(2) Scanning speed of radar antenna;

(3) Beam width of radar antenna;

(4) Repetition frequency of radar pulse;

(5) Initial position of radar antenna;

Owing to different radars exist differences in the five aspects above, beam cannot observe the object and echo pulse cannot be synchronized in the process of neighboring station-distributed radar observing, which lead to time asynchronous of echo for calculating cross cumulant, and echo without the maximize correlation. Moreover, it will impact on the signal to clutter ratio output of the domain of dependence and go against target detection.

When bistatic radar is neighboring station-distributed, time difference of radar antenna passing north $\Delta T_{1}$ can be gained through measuring moment of two radars passing true north; if assume radar 1 antenna passing true north prior to radar 2 antenna, repetition frequency of radar 1 and radar 2 are $f r_{1}, f r_{2}$ respectively and apply pulse Doppler system, then in time difference $\Delta T_{1}$, number of radar 2 transmission pulse is:

$$
\Delta N=\frac{\Delta T_{1}}{T r_{2}}
$$

It is observed that pulse periods of two radars echo will not be corresponded due to existing of $\Delta T_{1}$. Therefore, beam synchronization can be realized by seeking out the first echo pulse of radar 1 passing north and corresponding echo pulse of radar 2 on the basis of $\Delta N$.

At this point, two radars have completed beam alignment and approximately simultaneous observation object. As a result of the differences of beam width, antenna scanning period as well as repetition period, echo pulses of two radars cannot be one-to-one corresponded yet, and it is still required to conduct impulsive synchronizing processing.

Obviously, it is feasible to select radar with less number of pulses as a benchmark for synchronization. In order to analyze conveniently, assume that radar 1 has less number of pulses If the number of transmission pulse is $N$ when antenna scanning a circle, then it can be considered as that the entire scanning area is divided into $\mathrm{N}$ sections. Antenna beam will transmit and receive one pulse when rotating to every section and interval is the repetition period of radar pulse. In this case, corresponding relationship of radar 1 and radar 2's corresponding pulse can be obtained in accordance with radar parameters, namely:

(1) Echo pulse sequence number of radar 1

(2) Echo pulse sequence number of radar 2

$$
\operatorname{fix}\left(\frac{\Delta T_{1}+(n-1) T r_{1}}{T r_{2}}\right)
$$


Hereinto, fix(.) represents roundness;

After the processing mentioned above, two radars under neighboring station distribution complete the processing of beam alignment and pulse synchronization that basically achieve effectiveness of simultaneous observation to the same area. In addition, its echo pulse can be used for cross cumulant calculation, and subsequent testing etc.

\section{Simulation analysis}

This paper applies regular zero memory nonlinearity method to simulate clutter environment, and adopts amplitude statistical distribution model in lines with Rayleigh distribution and K-distributed because that the majority of low altitude target's echo interference is land and sea clutter. Furthermore, power spectrum model is complied with Gaussian spectrum clutter model so as to better match with the land and sea clutter environment. Band width of this clutter is $3 \mathrm{~km}$, and located within a certain range of $[13.5 \mathrm{Km}, 16.5 \mathrm{Km}]$ from radar; carrier frequencies of two radars are $2 \mathrm{GHz}$ and $4 \mathrm{GHz}$, band width of radar is set as $100 \mathrm{MHz}$, pulse width is $100 \mathrm{~ms}$, repetition frequency of pulse is $2000 \mathrm{~Hz}$, sampling rate is $200 \mathrm{MHz}$, number of echo pulse is 10 , and signal form adopts regular chirp signal; object is assumed as point of zero velocity, and distance between the object and the radar is $16 \mathrm{~km}$; length between cross cumulant and sliding window is set as 500 .
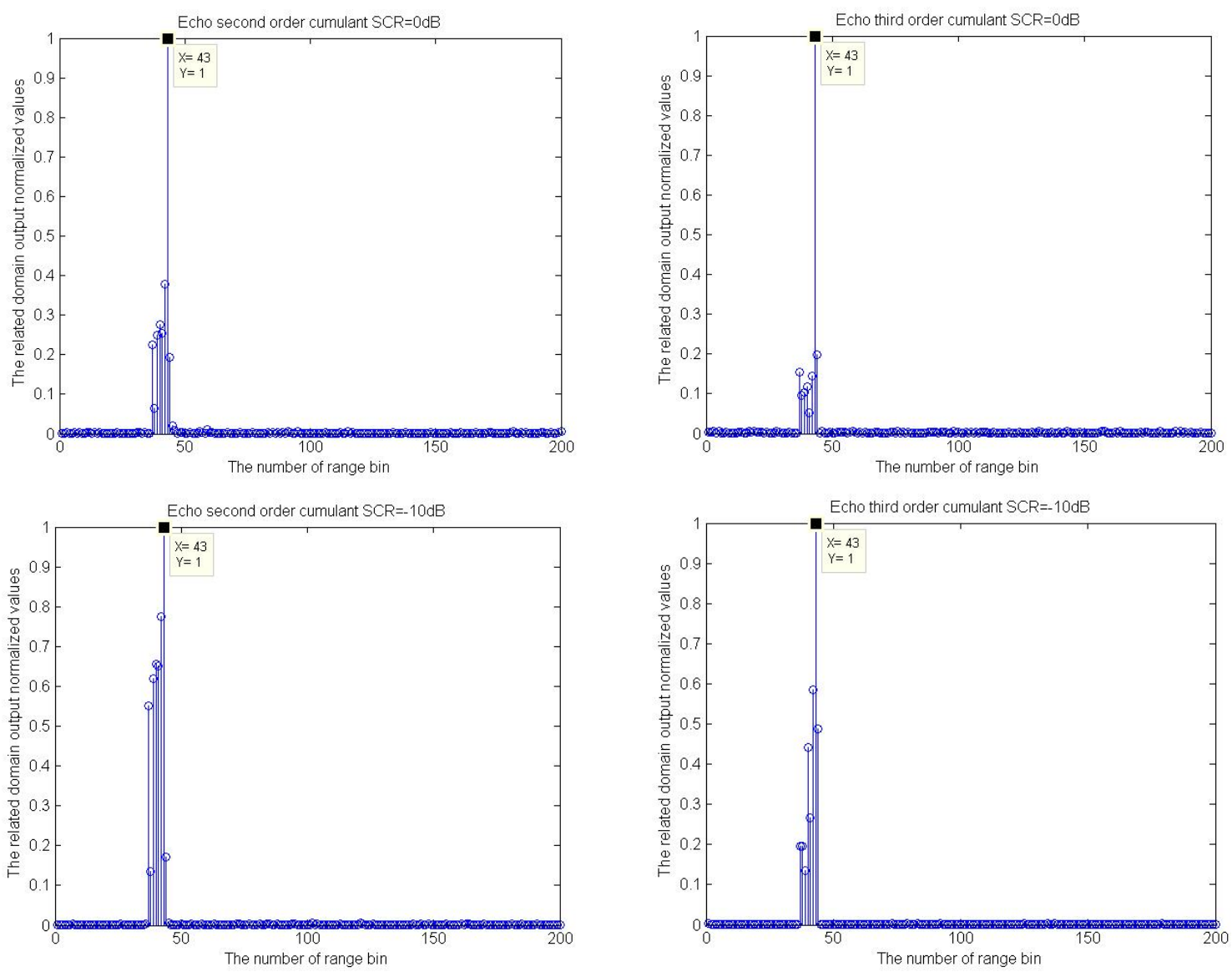

Fig. 1 The related domain output when SCR=0db and SCR=-10dB

The figure.1 shown at neighboring station-distributed condition, indicates fusion processing results of second-order and third-order cross cumulant under Rayleigh distribution clutter environments, when signal to clutter ratio of input signal is $0 \mathrm{~dB}$ and $-10 \mathrm{~dB}$; It can be found that the $43^{\text {rd }}$ range bin, namely the place of the object, has significantly higher cross-cumulant output than other range bins, and at the same time the fused result of third-order cross cumulant is superior to the fused result of second-order cross cumulant because that echo pulse sample will be increased along with increase of order so as to enhance statistical energy and output effect of domain of dependence, whereas calculated amount will be increased as well. 


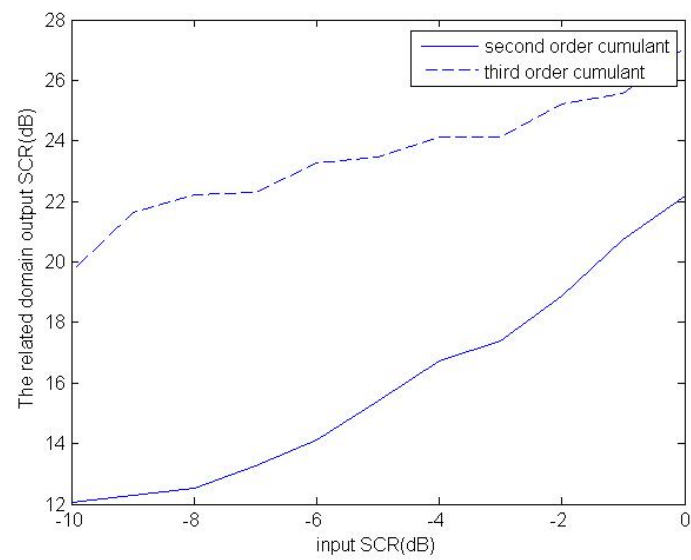

Fig. 2 The related domain output SCR when input SCR is changing

The figure. 2 is a change chart that indicates the signal to clutter ratio of second-order and third-order cross-cumulant's result output is changed along with the signal to clutter ratio of input. As can be seen from the figure, third-order cross cumulant is able to bring a better improvement of signal to clutter ratio than second-order cross cumulant that is in accord with the theory, and signal to clutter ratio of second-order cross cumulant's result output decreases faster than third-order following reduce of input signal to clutter ratio. In conclusion, the processing efficiency of third-order cross cumulant is better than the second-order cross cumulant, while both of them are capable of significantly improving signal to clutter ratio of output signal.

\section{Summary}

With the development of the war, the conventional single radar units already more and more difficult to meet the demand for low-altitude and slow-speed dim target detection, this paper considers the use of clutter under different carrier frequency echo correlation weak correlation stronger the characteristics of the target, simulated clutter environment, is commonly used by computing two corresponding pulse radar related domain output, the purpose of to improve signal to noise ratio is beneficial to the follow-up for low-altitude and slow-speed dim target detection .

\section{References}

[1]Huabang Zhu,Juan Du. “Four threats”survival and combat environment radar technology analysis [J].Aerodynamic Missile ,2005,01:61-64.

[2]Chong Wu,Haifei Li,Hong Wang,Jianbin Li.The analysis of PD radar detecting low-altitude and slow-speed dim target performance [J].Science Technology and Engineering,2013,28:8492-8496.

[3]Jinjun Ma,Weijun Ma,Mingbo Zhao,Kaifeng Zhang.Low Altitude/very low altitude penetration and radar countermeasures[J]. National Defense Science \& Technology,,2011,03:26-35.

[4]Hanbin Hua. Study on Characteristics and Critical Technologics of Radar Networking [J]. Modern Electronics Technique,2007,23:33-35.

[5]Shyu H C,Sun Y S,Shen W H.The analysis of scan-to-scan integration technique for sea clutter[C].Proceedings of the 1994 IEEE National Radar Conference Atlanta, GA 1994,228-233.

[6]Cheng Yin,Zhiming Wu,Huaiqun Deng Application of high-order statistics in seismic exploration [J]. Progress In Geophysics,2003,03:546-550. 